Int. J. Morphol.,

33(3):1126-1129, 2015.

\title{
¿Músculo Plantar?
}

\author{
Plantaris Muscle?
}

Rafael Coello ${ }^{*, * *}$; Pamela Carvallo ${ }^{*, * * * *}$; Eugenia Carvallo ${ }^{*, * * * * * *} \&$ Mariano del Sol $^{* * * * *, * * * * * * * * *}$

COELlO, R.; CARVAllo, P.; CARVALlO, E. \& DEL SOL, M. ¿Músculo plantar? Int. J. Morphol., 33(3):1126-1129, 2015.

RESUMEN: Todos los campos científicos de la salud utilizan Terminologia Anatomica como herramienta de comunicación para las descripciones en artículos científicos impresos y electrónicos; las denominaciones anatómicas humanas son el punto de partida para ordenar todo idioma científico médico respetando sus raíces latinas y griegas; sin embargo, existen términos que se originaron por simbolismos, respetando ideografías y otros producto de la disección animal. Se revisará la denominación de músculo plantar (Musculus plantaris) al músculo ubicado en el compartimiento posterior de la pierna que consta en la Nomina Anatomica editada en Basilea en 1895 y en el texto Terminologia Anatomica publicada en 1998, documento oficial aceptado por la FIPAT; la semántica y las características descriptiva del término músculo plantar implican su ubicación en el pie pero su identidad morfológica demuestra una inconsistencia nominativa por presentar origen e inserción que corresponden a la pierna; se sugiere revisar su denominación de músculo plantar y considerar su cambio por músculo femorocalcáneo (Musculus femorocalcaneous).

PAlABRAS ClAVE: Músculo Plantar; Terminologia Anatomica; Programa Federativo Internacional de Terminología Anatómica (FIPAT); Músculo Femorocalcáneo.

\section{INTRODUCCIÓN}

Desde la Nomina Anatomica de Basilea (His, 1895) se decidió unificar y armonizar el lenguaje anatómico, aspecto que ha preocupado a la comunidad científica permanentemente durante diecisiete Congresos Internacionales de Anatomía realizados hasta la presente. Durante el XIII Congreso celebrado en Rio de Janeiro en 1989 se constituyó la FCTA (Comité Federativo de Terminología Anatómica) que elaboró el último texto de Terminologia Anatomica en cooperación con la Federación Internacional de Asociaciones de Anatomía (IFAA), texto que ofrece la versión oficial de los términos anatómicos (FICAT).

La denominación de "músculo plantar" es el término aceptado a dicho músculo por el texto oficial de Terminologia Anatomica divulgada por el Programa Federativo Internacional de Terminología Anatómica (FIPAT por sus siglas en inglés) y aprobado por la Federación Internacional de Asociaciones de Anatomía (IFAA por sus siglas en inglés) difundida desde 1998 en latín e inglés, texto traducido al español el
2001. El músculo plantar junto a los músculos gastrocnemio y sóleo ocupan el plano superficial del compartimiento posterior de la pierna (Moore \& Dalley, 2001), tiene origen en la región femoral posterior e inserción en el hueso calcáneo por lo que se estima su nombre no correspondería a su denominación, aspecto que se analizará en esta comunicación.

En Terminologia Anatomica se enlistan los términos en tres idiomas (latín, inglés y español), encontrándose en la sección respectiva la denominación del músculo plantar de esta manera: (A04.7.02.049) Musculus plantaris, plantaris muscle, músculo plantar (FICAT, 2001; FIPAT, 2011); del mismo modo aparece en publicaciones relacionadas. En los textos de morfología y publicaciones científicas que utilizan convencionalmente Terminologia Anatomica se puede encontrar denominaciones parecidas; se usa universalmente el nombre del músculo plantar en la bibliografía electrónica y es utilizado convencionalmente en los centros educativos de Ciencias de la Salud.

* Universidad de Guayaquil, Guayaquil, Ecuador.

** Doctorado en Ciencias Morfológicas, Universidad de La Frontera, Temuco, Chile.

**** Universidad de Católica de Temuco, Temuco, Chile.

***** Universidad Mayor, Temuco, Chile.

****** Facultad de Medicina, Centro de Excelencia en Estudios Morfológicos y Quirúrgicos (CEMyQ), Universidad de La Frontera, Temuco, Chile.

******* Center for Biomedical Research, Universidad Autónoma de Chile, Temuco, Chile. 
En el Tratado de Anatomía Humana de Sapey en 1854, se lo indicaba como músculo plantar delgado; Petréquin en 1868 menciona en su texto Tratado de Anatomía Topográfica Médico Quirúrgico el "pequeño femorocalcaniano" al referirse al músculo plantar delgado que el autor sugiere llamarlo mejor "mús-

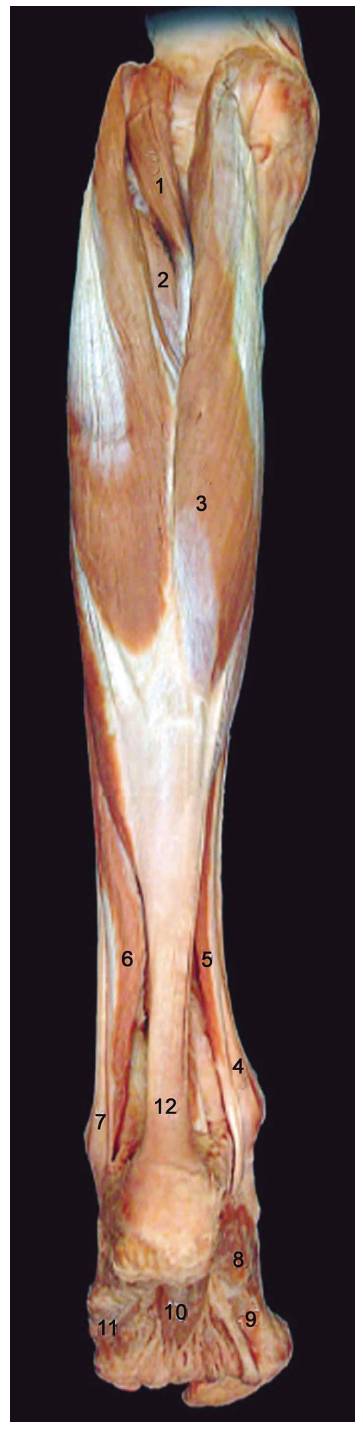

Fig. 1. Visión posterior y superficial de la pierna. 1.M.plantar; 2. M. tibial posterior; 3. M. gastrocnemio; 4. M. flexor largo de los dedos; 5. M.flexor largo del hállux; 6. M.sóleo; 7. Tendón fibular largo; 8. M. abductor del hállux; 9. M. flexor corto del hállux; 10. M.flexor corto de los dedos; 11. M. abductor dedo mínimo.

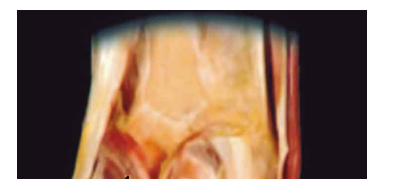

logía rudimentaria y con muchas variaciones (doble unilateralmente, duplicidad, ausencia y otros le señalan escasa importancia clínica); pero todos los autores reiteran que a pesar de sus variaciones el músculo tiene origen femoral e inserción calcánea. Otras series demuestran que el músculo plantar se inserta en distintas ubicaciones; Le Double (1897) reporta múltiples inserciones incluyendo unas en menor proporción y con escasas fibras en la aponeurosis plantar sin cambiar su origen en el cóndilo lateral del fémur; finalmente se reconoce que en aproximadamente el 7\% de la población está ausente. Se estima necesario analizar la denominación del músculo por su presencia en la pierna y ausencia total en el pie en el humano.

\section{FUNDAMENTACIÓN}

Los problemas lexicológicos y lexicográficos anatómicos han sido enfrentados permanentemente en reuniones regionales como el Simposio Iberolatinoamericano de Terminología Anatómica (SILAT) (Losardo et al., 2010), organismo encargado de realizar revisiones por expertos en secuenciales reuniones que se han realizado periódicamente en forma bianual desde el 2009.

Por razones idiomáticas, Terminologia Anatomica acepta incorporaciones o modificaciones en el listado plenamente justificadas; los términos deben ser sencillos, descriptivos, precisos e informativos. El actual Programa Federativo Internacional de Terminología Anatómica (FIPAT), organismo que sucedió a la FICAT y a la FCAT ha ratificado las denominaciones; para una incorporación se admiten tanto el análisis proveniente de grupos de trabajo, sugerencias individuales o por medio del foro online que presenta la FIPAT.

Fig. 2. Visión posterior y profunda de la pierna.1. M. gastrocnemio; 2. M. plantar; 3. M. poplíteo; 4. M. soleo; 5. M. tibial posterior; 6. M. flexor largo de los dedos; 7. M. flexor largo del hállux; 8. M. fibular corto; 9. M. fibular largo; 10. Fibula.

Los textos anatómicos clásicos (Testut \& Latarjet, 1979; Rouvière \& Delmas, 2000) describen al músculo plantar con sus orígenes en el cóndilo lateral del fémur y sus inserciones calcáneas. Estudios en series ratifican esta descripción indicando que el tendón se dirige hacia inferomedial entre el músculo gastrocnemio y el músculo sóleo; su inserción puede ser en el tendón calcáneo o en otro sitio de la superficie medial del hueso calcáneo (Feneis \& Dauber, 2000; Kwinter et al., 2010); también se mencionan muchas variaciones en toda su morfología: dobles músculos plantares unilaterales (Kwinter et al.) doble origen e inserción única (Soni et $a l ., 2014$ ) ausencias en porcentajes variables (Testut, 1884; Venter 
et al., 2011) y comportamientos discordantes del componente muscular con la parte fibrosa (Aragão et al., 2010).

Sus inserciones tienen distintos destinos: a) Tejido celular entre los músculos gastrocnemios y sóleo; b) Superficie medial del tendón calcáneo; c) Superficie posterior del tendón calcáneo; d) Bolsa serosa entre el tendón calcáneo y el hueso; e) Tejido fibroadiposo del tendón calcáneo; f) Aponeurosis plantar (Le Double). En un estudio con 46 muestras (Fredman et al., 2010) establecieron lo siguiente: disposición estándar $(56,52 \%)$, estaba presente pero con variaciones $(30,44 \%)$ y estaba ausente $(13,04 \%)$; por su parte Nayak et al. (2010) en una serie de 52 muestras encontró más variaciones tanto en el origen como en la inserción.

Sus funciones de flexión de la rodilla y extensión de la articulación talocrural en el hombre no tienen relación con la prensión y la acción de trepar que cumple en algunos primates, de hecho en el hombre la denominación de músculo plantar implica un rasgo aparentemente vestigial (Kwinter et al.), esto convalida que su ubicación topográfica en humanos es un detalle válido para cambiar su denominación, aceptándose que participa trivialmente en las actividades ambulatorias.

Dentro de sus limitadas funciones, bien puede ser utilizado como injerto en lesiones tendinosas sin afectar al donante (Dos Santos et al., 2009), por tal razón estudios en distintas poblaciones se preocupan de establecer las longitudes de la porción muscular y del componente tendinoso (Venter et al.).

Para las teorías evolucionistas el músculo plantar es un elemento vestigial como resultado de la acción trepadora de algunos mamíferos y antropoides llegando en algunos de ellos su inserción hasta la aponeurosis plantar (Infestas et al., 2012); en el humano, el músculo plantar se estudia como un componente del grupo muscular del compartimiento pos- terior de la pierna, descrito así en todas las publicaciones biomédicas; incluso sus variaciones se estiman en aproximadamente un $10 \%$ (Suvagasi et al., 2010) lo que implica que en el hombre no tiene la importancia que tiene en el primate. Por otro lado, todas las publicaciones han utilizado de manera constante el término músculo plantar en sus descripciones, dejando implícito en forma tácita su aceptación a la denominación del músculo como parte del pie sin alcanzar su planta, esto ha motivado el presente documento.

Finalmente, la Real Academia de Lengua española (RAE) acepta el término plantar (del latín plantaris) (en su acepción anatómica) "a todo aquello perteneciente o relativo a la región plantar". Sus definiciones latinas incluyen los mismos significados (Plantar: Nominativo de 3ra declinación cuyo significado es "planta del pie"). La denominación Músculo Femorocalcáneo (Musculus femorocalcaneous) se ajusta a los requerimientos de la FIPAT por contener la descripción e información más adecuada para el músculo respetando sus orígenes e inserciones sin desmedro de aceptar sus variaciones dentro de estos reparos anatómicos.

\section{CONCLUSIÓN}

La Terminologia Anatomica Humana utilizada en la comunidad científica debe ser precisa, simple e informativa para que cumpla su rol lexicográfico exacto (Didio, 1998); la denominación de músculo plantar en latín, inglés y español conlleva una definición y semánticas relacionadas con el pie, lo que puede confundir en medicina deportiva, ortopedia, cirugía, antropología y en medicina física. La presente revisión pretende que se analice la posibilidad de cambiar el término músculo plantar por músculo femorocalcáneo que cumple con las directrices de Terminologia Anatomica por ser descriptivo y corresponder a su origen, inserción y ubicación topográfica.

COEllo, R.; CARVAllo, P.; CARVAllo, E. \& DEL SOL, M. Plantaris muscle? Int. J. Morphol., 33(3):1126-1129, 2015.

SUMMARY: All fields in the health sciences use anatomical terminology as a communication tool for the descriptions in print and electronic scientific articles; human anatomical names are the starting point for organizing all scientific medical language respecting their Latin and Greek roots. However, there are terms that originated by symbolism, respecting ideographs, the result of animal dissections. The name plantar muscle (musculus plantaris) will be reviewed as the muscle located in the posterior compartment of the leg as it appears in the Nomina Anatomica or BNA published in Basel in 1895 and Anatomical Terminology text published in 1998, an official document accepted by the FIPAT. Semantic and descriptive characteristics of the term imply the location plantaris muscle in the foot but its morphological identity shows inconsistency by presenting origin and insertion corresponding to the leg. A review of the name is suggested and that the change of plantar muscle to Femorocalcaneo (femorocalcaneous musculus) be considered.

KEY WORDS: Plantar muscle; Terminologia Anatomica (TA); Federative International Programme for Anatomical Terminology a (FIPAT); Femorocalcaneous muscle. 


\section{REFERENCIAS BIBLIOGRÁFICAS}

Aragão, J. A.; Reis, F. P.; Guerra, D. R. \& Cabral, R. H. The occurrence of the plantaris muscle and its muscle-tendon relationship in adult human cadavers. Int. J. Morphol., $28(1): 255-8,2010$.

Didio, L. J. A. History of International Anatomical Terminology. In: Federative International Program on Anatomical Terminologies (FICAT). Terminologia Anatomica. International Anatomical Terminology. Stuttgart, Thieme, 1998.

Dos Santos, M. A.; Bertelli, J. A.; Kechele, P. R. \& Duarte, H. Anatomical study of the plantaris tendon: reliability as a tendoosseous graft. Surg. Radiol. Anat., 31(1):59-61, 2009.

Federative International Committee on Anatomical Terminology (FICAT). Terminologia Anatomica. International Anatomical terminology. Stuttgart, Georg Thieme Verlag, 2001.

Federative International Programme on Anatomical Terminologies (FIPAT). Terminologia Anatomica. International Anatomical terminology. 2nd ed. Stuttgart, Georg Thieme Verlag, 2011.

Feneis, H. \& Dauber, W. Pocket Atlas of Human Anatomy. Based on the International Nomenclature. 4th ed. Stuttgart, Thieme, 2000.

Freeman, A. J.; Jacobson, N. A. \& Fogg, Q. A. Anatomical variations of the plantaris muscle and a potential role in patellofemoral pain syndrome. Clin. Anat., 21(2):178-81, 2008.

Gray, H. \& Lewis, W. H. Anatomy of the Human Body. 20th ed. Philadelphia, Lea \& Febiger, 1918.

His, W. Nomina Anatomica. Leipzig, Verlag von Veit \& Comp., 1895.

Infestas, E.; Pastor, J. F.; Diogo, R. \& Wood, B. Comparative anatomy of the lower limb muscles of hominoids: attachments, relative weights, innervation, functional morphology and evolution. Am. J. Phys. Anthropol., 147(Suppl. 54):172, 2012.

Kwinter, D. M.; Lagrew, J. P.; Kretzer, J.; Lawrence, C.; Malik, D.; Mater, M. \& Brueckner, J. K. Unilateral double plantaris muscle: a rare anatomical variation. Int. J. Morphol., 28(4):1097-9, 2010.

Le Double, F. Traité des variations du Systeme Musculaire de l'Homme. Paris, Ed. Schleicher Fréres, 1897. T II.

Losardo, R. J.; Cruz Gutiérrez, R.; Rodríguez Torres, A.; Prates, J. C. \& Prates, N. E. V. B. Iberia-Latin-American Symposia of Morphological Terminology (SILAT). First two years and statute. Int. J. Morphol., 28(4):1323-6, 2010.
Moore, K. L. \& Dalley, A. F. Anatomia con Orientación Clinica. $4^{\mathrm{a}}$ ed. Buenos Aires, Editorial Médica Panamericana, 2001. pp.519-21.

Nayak, S. R.; Krishnamurthy, A.; Ramanathan, L.; Ranade, A. V.; Prabhu, L. V.; Jiji, P. J.; Rai, R.; Chettiar, G. K. \& Potu, B. K. Anatomy of plantaris muscle: a study in adult Indians. Clin. Ter., 161(3):249-52, 2010.

Rouvière, H. \& Delmas, A. Anatomía Humana: Descriptiva, topográfica y funcional. $10^{\mathrm{a}} \mathrm{ed}$. Barcelona, Masson, 2002. V.3.

Soni, S.; Saxena, A.; Ghulyani, T. \& Rani-Das, A. A biceps plantaris in the popliteal region - case report. Eur. J. Anat., 18(1):32-3, 2014.

Sugavasi, R.; Latha, K.; Devi, Indira; Jetti, R.; Sirasanagandla, S. R. \& Gorantla, V. R. A case report of variant insertion of plantaris muscle and its morphological and clinical implications. J. Morphol. Sci., 30(4):304-5, 2013.

Testut, L. Anomalies musculaires chez l'Homme. Paris, Masson, 1884.

Testut, L. \& Latarjet, A. Tratado de Anatomía Topográfica. 8a Ed. Barcelona, Salvat, 1979. TII. pp.1153-4.

Venter, G.; van Schoor, A. \& Bosman, M. C. Clinical applications of the plantaris muscle in reconstructive surgery. Clin. Anat., 24(7):924, 2011.

Dirección para Correspondencia:

Rafael Coello Cuntó

Universidad de Guayaquil

Guayaquil

ECUADOR

Email: msdracocu@hotmail.com

Recibido : 11-04-2015

Aceptado: 17-06-2015 